\title{
Temperature-carrier-concentration phase diagram of a two-dimensional doped $d$-wave superconductor
}

\author{
V.M. Loktev \\ Bogolyubov Institute for Theoretical Physics of the National Academy of Sciences of Ukraine \\ 14-b, Metrologicheskaya Str. Kiev, 03143, Ukraine \\ E-mail: vloktev@bitp.kiev.ua
}

V.M. Turkowski

Department of Physics, Georgetown University, Washington, D.C. 20057, USA

E-mail: turk@physics.georgetown.edu

Received March 1, 2006

\begin{abstract}
The finite-temperature properties of a two-dimensional $d$-wave superconductor with the Lifshitz disorder, introduced by dopants, are studied. The doping dependence of the mean-field critical temperature $T_{C}^{M F}$ and of the superconducting critical temperature $T_{C}$ defined by the Berezinskii - Kosterlitz - Thouless transition are calculated at different values of coupling, dopant potential, and intermediate boson energy. It is shown that superconductivity tends to disappear with increasing doping when the dopant potential is large enough, though the metallic properties of the system are preserved.
\end{abstract}

PACS: 74.20.Rp, 74.25.Dw, 74.40.+k, 74.62.Dh, 74.72.-h

Keywords: two-dimensional $d$-wave superconductor.

\section{Introduction}

It is known that the high-temperature superconductors (HTSCs) in the non-doped regime are antiferromagnetic insulators (or semiconductors, more precisely), similarly to many other transition metal oxides, with dielectric gap $\simeq 2 \mathrm{eV}$ (see, for example reviews [1-4]). Free carriers (electrons or holes) can be introduced into the conduction band by changing temperature or pressure or by injecting some donor or acceptor impurities. The last possibility is the most effective way to transform the layered copper oxide insulators into metals, which exhibit the high-temperature superconductivity. Since, in principle, different valence dopants can be introduced into the system in any proportion, the physical properties of HTSC compounds are studied as a function of dopant concentration $x$, or equivalently as a function of carrier concentration c. It should be noted, however, that $\mathrm{YBa}_{2} \mathrm{Cu}_{4} \mathrm{O}_{8}$ is probably the unique HTSC cuprate compound with the metallic phase in its initial stoichiometric state. This doesn't allow one to study the effects of doping in this compound.

Despite the fact that the quantities $x$ and $c$ can be considered equal (or proportional to each other, as in the cuprates with many layers), the effects of carriers and dopants on the material properties are quite different. Changing of the carrier density leads to a rather smooth evolution of the metallic, transport, and optical properties of HTSCs. At the same time, randomly distributed dopants [5] together with chaotic electric and deformation fields, which are always generated by them, play a role of carrier scatterers, or centers of localization. As a result, even clean HTSCs should be considered as «bad metals» due to the condition $c \simeq x \equiv c_{\text {dop }}$. The mean free path $l$ and the Fermi momentum $k_{F}$ in these compounds are related as $k_{F} l \sim 1$, and the number of the impurity centers (including external ones) cannot be smaller than the carrier number, contrary to the cases of the conventional metals and superconductors. In fact, as it follows from the experiments, the doping initially leads to destruction of the long-range magnetic order in the dielectric phase. 
Then, the system gets properties of a doped semiconductor with increasing $x$ (or $c$ ), and it becomes a metal when $c$ is larger than the mobility threshold value $c_{\text {met }}$.

The effect of impurities on the properties of usual (low-temperature) $s$-wave superconductors was established a long time ago. Nonmagnetic impurities with small concentration have practically no effect on the superconducting properties (in particular, they hardly change the critical temperature $T_{c}[9]$ ), and their role is constrained to make the superconducting gap more isotropic. In contrast, magnetic impurities result in a fast suppression of $T_{c}$, when their concentration is increasing [10]. Impurities are external objects in both cases, and their concentration is not connected with charge carrier concentration. It is important that the superconducting condensate in ordinary superconductors is formed and exists without any doping.

This difference is principal, since practically in all cases the doping is necessary for metalization of the cuprate materials. Therefore the role of the doping is «constructive». On the other hand, at densities equal to the carrier density, the presence of the dopants can cause suppression of superconductivity, similarly to external magnetic impurities in usual superconductors. Moreover, since both magnetic and nonmagnetic external impurities change $T_{c}$ in the HTSCs significantly (see [11-16] and also review [4]), this question becomes even more interesting. A zero-temperature self-consistent study of this two-fold role of the dopants in the case of a two-dimensional (2D) system with the $s$-wave pairing demonstrates [17] that superconductivity exists only in small concentration region $c_{\text {met }}<c<c_{\text {sup }}$, where $c_{\text {sup }}$ is the concentration value above which superconductivity is suppressed [17]. This quantity is defined by the condition of equality of the inverse lifetime of a pair $\tau_{\text {pair }}^{-1}$ and superconducting gap $\Delta_{s}: \Delta_{s} \tau_{\text {pair }} \sim \hbar$. The value of $\tau_{\text {pair }}$ is defined by rate of the scattering of pairs on local inhomogeneites, observed in HTSCs [18].

The zero-temperature superconducting properties of a 2D model with the doping induced by the Lifshitz disorder in the case of a $d$-wave pairing were studied in $[19,20]$. The corresponding finite temperature problem is complicated by the fact that a two-dimensional system with a continuous symmetry of the order parameter can't have a long-range order at finite temperatures. Due to strong order parameter fluctuations only the phase with algebraically correlated order parameter is possible in this case, even when the system has no impurities. The critical temperature of this transition is usually called the Berezinskii-Kosterlitz-Thouless (BKT) transition temperature (for review, see [21]), but we shall identify it with $T_{C}$ below. Therefore, the temperature-carrier-density phase diagram of the $2 \mathrm{D}$ doped superconducting metals consists of three qualitatively different regions:

i) $T>T_{c}^{M F}(c)$, when the superconductivity is absent (normal phase);

ii) $T_{c}(c)<T<T_{c}^{M F}(c)$, when the order parameter already exists, but its phase correlations rapidly (exponentially) decay with distance. This phase is often interpreted as the pseudogap phase of HTSCs;

iii) $T<T_{c}(c)$, when the system demonstrates a quasi-long-range superconducting order with an algebraically correlated order parameter.

This phase diagram was studied in cases with different kinds of electron attraction. For example, it was considered in the case of a model with the local attraction in [22] (see also a recent paper [23]), and with a phonon-exchange attraction in $([24,25]$ ) (for review see [21]). It is important to understand how the disorder affects the critical temperature $T_{c}$ in the $2 \mathrm{D}$ system at different values of doping.

Below we make an attempt to study self-consistently the superconducting temperature-carrier density phase diagram of the system with a boson-mediated attraction in the presence of the Lifshitz disorder, which is introduced by doping into the initially insulating system.

It is a great pleasure and honor for us to contribute this special issue of Low Temperature Physics in memory of Prof. B.G. Lazarev, an outstanding low-temperature and solid state physicist, whose works led to a deeper understanding of the physics of normal and superconducting metals, especially mechanisms of the behavior of these systems in the presence of strong external fields and pressure.

\section{The main equations}

The Hamiltonian of the 2D fermion system with a boson-exchange interaction and in the presence of a disorder can be written as:

$$
\begin{gathered}
H(\tau)=-\sum_{\mathbf{n}, \mathbf{m}, \sigma} t_{\mathbf{n m}} a_{\mathbf{n} \sigma}^{\dagger}(\tau) a_{\mathbf{m} \sigma}(\tau)+ \\
+\left(\frac{W}{2}-\mu\right) \sum_{\mathbf{n}, \sigma} a_{\mathbf{n} \sigma}^{\dagger}(\tau) a_{\mathbf{n} \sigma}(\tau)- \\
-\frac{1}{2} \sum_{\mathbf{n}, \mathbf{m}, \sigma} a_{\mathbf{n}}^{\dagger} \sigma(\tau) a_{\mathbf{m} \sigma}^{\dagger}(\tau) V_{\mathbf{n m}} a_{\mathbf{m} \sigma}(\tau) a_{\mathbf{n} \sigma}(\tau)- \\
-\frac{V_{L}}{N} \sum_{\mathbf{n}, \sigma} a_{\mathbf{n} \sigma}^{\dagger} a_{\mathbf{n} \sigma},
\end{gathered}
$$

where $a_{\mathbf{n} \sigma}^{\dagger}(\tau), a_{\mathbf{n} \sigma}(\tau)$ are creation and annihilation operators of fermion with spin $\sigma=\uparrow, \downarrow$ on site $\mathbf{n}$; $\tau$ is the imaginary time; $t_{\mathrm{nm}}$ is the nearest neighbor hopping operator. The dispersion relation of the free elec- 
trons on the square lattice can be chosen in the simplest form: $\xi_{\mathbf{k}}=4 t-2 t\left[\cos \left(k_{x} a\right)+\cos \left(k_{y} a\right)\right]-\mu$ with the bandwidth $W=8 t$, where $t$ is the nearest neighbor hopping parameter; $\mu$ is the chemical potential. The interaction potential $V_{\mathrm{nm}}$ in (1) corresponds to the potential $V_{\mathbf{k q}}$ in the momentum space. We shall consider the $d$-wave pairing case, which can be generated by the potential $V_{\mathbf{k q}}=V \gamma_{\mathbf{k}} \gamma_{\mathbf{q}}$, where $\gamma_{\mathbf{k}}=$ $=\left[\cos \left(k_{x} a\right)-\cos \left(k_{y} a\right)\right] \theta\left(\varepsilon_{D}^{2}-\xi_{\mathbf{k}}^{2}\right)$. The theta-function guarantees that the quasiparticle with the energies in the $\left\langle\mathrm{BCS} \gg\right.$ belt $\varepsilon_{D}$ around the Fermi level interact with each other $\left(\varepsilon_{D}\right.$ is the maximal energy of the intermediate bosons, which lead to the interparticle attraction); $V_{L}$ is an one-site impurity (here-dopant) potential. It corresponds to a ran- domly distributed shift of the on-site energy, according to the Lifshitz model of the disorder $[26,27]$.

In order to study properties of the system, it is convenient to derive the thermodynamic potential. It can be calculated by evaluating the partition function of the system. This function can be written as

$Z=\int D a^{\dagger} D a \exp \left\{-\int_{0}^{\beta} d \tau\left[\sum_{\mathbf{n}, \boldsymbol{\sigma}} a_{\mathbf{n} \sigma}^{\dagger}(\tau) \hat{\partial}_{\tau} a_{\mathbf{n} \boldsymbol{\sigma}}(\tau)+H(\tau)\right]\right\}$.

The Hubbard-Stratonovich transformation with bilocal fields $\varphi_{\mathrm{nm}}(\tau)$ and $\varphi_{\mathrm{nm}}^{\dagger}(\tau)$ can be applied to the nonlinear term in the partition function (2):

$$
\begin{gathered}
\exp \left[\int_{0}^{\beta} d \tau \sum_{\mathbf{n}, \mathbf{m}} a_{\mathbf{n} \uparrow}^{\dagger}(\tau) a_{\mathbf{m} \downarrow}^{\dagger}(\tau) V_{\mathbf{n m}} a_{\mathbf{m} \downarrow}(\tau) a_{\mathbf{n} \uparrow}(\tau)\right]= \\
=\int D \varphi^{\dagger} D \varphi \exp \left[-\int_{0}^{\beta} d \tau \sum_{\mathbf{n}, \mathbf{m}}\left(\frac{\left|\varphi_{\mathbf{n m}}(\tau)\right|^{2}}{V_{\mathbf{n m}}}-\varphi_{\mathbf{n m}}^{\dagger}(\tau) a_{\mathbf{n} \downarrow}(\tau) a_{\mathbf{m} \uparrow}(\tau)-a_{\mathbf{n} \uparrow}^{\dagger}(\tau) a_{\mathbf{m} \downarrow}^{\dagger}(\tau) \varphi_{\mathbf{n m}}(\tau)\right)\right] .
\end{gathered}
$$

The complex order parameter can be expressed as $\varphi_{\mathrm{nm}}(\tau)=\Delta_{\mathrm{nm}}(\tau) \exp \left(i \theta_{\mathrm{nm}}(\tau)\right)$, where $\Delta_{\mathrm{nm}}(\tau)$ is the modulus and $\theta_{\mathbf{n m}}(\tau)$ is the phase of the order parameter. We assume that $\Delta_{\mathrm{nm}}(\tau)$ depends on the relative coordinate of the operators $\mathbf{r}=\mathbf{R}_{\mathbf{n}}-\mathbf{R}_{\mathbf{m}}$ only, and $\theta_{\mathbf{n m}}(\tau)$ depends only on the center of mass coordinate $\mathbf{R}=\left(\mathbf{R}_{\mathbf{n}}+\mathbf{R}_{\mathbf{m}}\right) / 2: \quad \varphi_{\mathbf{n m}}(\tau) \simeq \Delta(\tau, \mathbf{r}) \exp (i \theta(\tau, \mathbf{R}))$. It is easy to understand this approximation in the following way. The Cooper pair dynamics is described by the modulus of the order parameter, and its space symmetry depends on the relative pair coordinate. On the other hand, the motion of the superconducting condensate is described by the phase of the order parameter, which changes slowly in space, and it can be described by the center of mass coordinate.
In order to evaluate the partition function of the system, it is convenient to introduce the two-component Nambu spinors

$$
\Psi_{\mathbf{n}}(\tau)=\left(\begin{array}{c}
a_{\mathbf{n} \uparrow}(\tau) \\
a_{\mathbf{n} \downarrow}^{\dagger}(\tau)
\end{array}\right), \quad \Psi_{\mathbf{n}}^{\dagger}(\tau)=\left(a_{\mathbf{n} \uparrow}^{\dagger}(\tau), a_{\mathbf{n} \downarrow}(\tau)\right),
$$

and to make the following decomposition of the fermion fields:

$$
\begin{gathered}
a_{\mathbf{n} \sigma}(\tau)=\chi_{\mathbf{n} \sigma}(\tau) \exp \left(i \theta_{\mathbf{n}}(\tau) / 2\right), \\
a_{\mathbf{n} \sigma}^{\dagger}(\tau)=\chi_{\mathbf{n} \sigma}^{\dagger}(\tau) \exp \left(-i \theta_{\mathbf{n}}(\tau) / 2\right)
\end{gathered}
$$

Then, in the continuum limit

$$
\begin{gathered}
\varphi_{\mathrm{nm}}^{\dagger}(\tau) a_{\mathbf{n} \downarrow}(\tau) a_{\mathrm{m} \uparrow}(\tau)+a_{\mathbf{n} \uparrow}^{\dagger}(\tau) a_{\mathrm{m} \downarrow}^{\dagger}(\tau) \varphi_{\mathrm{nm}}(\tau) \rightarrow \varphi_{\mathrm{nm}}^{\dagger} \Psi_{\mathbf{n}}^{\dagger}(\tau) \hat{\tau}_{-} \Psi_{\mathrm{m}}(\tau)+ \\
+\Psi_{\mathbf{n}}^{\dagger}(\tau) \hat{\tau}_{+} \Psi_{\mathbf{m}}(\tau) \varphi_{\mathrm{nm}}(\tau) \simeq \Delta\left(\tau, \mathbf{R}_{\mathbf{n}}-\mathbf{R}_{\mathbf{m}}\right) Y_{\mathbf{n}}^{\dagger}(\tau) \hat{\tau}_{1} \mathrm{Y}_{\mathrm{m}}(\tau)
\end{gathered}
$$

where $Y_{n}^{\dagger}(\tau)$ and $Y_{m}(\tau)$ are so called neutral Nambu spinor operators:

$$
Y_{\mathbf{n}}(\tau)=\left(\begin{array}{c}
\chi_{\mathbf{n} \uparrow}(\tau) \\
\chi_{\mathbf{n} \downarrow}^{\dagger}(\tau)
\end{array}\right), \quad Y_{m}^{\dagger}(\tau)=\left(\chi_{\mathbf{m} \uparrow}^{\dagger}(\tau), \chi_{\mathbf{m} \downarrow}(\tau)\right),
$$

and $\hat{\tau}_{ \pm}$are combinations of the Pauli matrices $\hat{\tau}_{1}$ and $\hat{\tau}_{2}: \hat{\tau}_{+}=\left(\hat{\tau}_{1} \pm \hat{\tau}_{2}\right) / 2$.

Substitution of (3) into (2) with approximation (4) allows one to obtain the expression for the parti- 
tion function after integration over the neutral Nambu spinors:

$$
Z=\int \Delta D \Delta D \theta \exp (-\beta \Omega(\Delta, \theta)),
$$

where the thermodynamic potential $\Omega(\Delta, \theta)$ has the following form:

$$
\beta \Omega(\Delta, \theta)=\int_{0}^{\beta} d \tau \int d^{2} r \frac{\Delta(\tau, \mathbf{r})^{2}}{V(\mathbf{r})}-\operatorname{Tr} \ln G^{-1} .
$$

The Green function for the Nambu spinors

$$
G_{\mathrm{nm}}\left(\tau_{1}, \tau_{2}\right)=\theta\left(\tau_{1}-\tau_{2}\right)<\Psi_{\mathbf{n}}\left(\tau_{1}\right) \Psi_{\mathrm{m}}^{\dagger}\left(\tau_{2}\right)>
$$

has the standard structure:

$$
G^{-1}=\mathcal{G}^{-1}-\Sigma,
$$

where $\mathcal{G}^{-1}$ is the part of the inverse Green's function that doesn't depend on the phase of the order parameter:

$$
\begin{aligned}
& \mathcal{G}^{-1_{\mathbf{n m}}^{-1}}\left(\tau_{1}, \tau_{2}\right)=\left\langle\tau_{1}, \mathbf{R}_{\mathbf{n}}\left|\mathcal{G}^{-1^{-1}}\right| \tau_{2}, \mathbf{R}_{\mathbf{m}}\right\rangle= \\
& =\delta_{\mathbf{n m}} \delta\left(\tau_{1}-\tau_{2}\right)\left[\partial_{\tau_{1}}-\hat{\tau}_{3}(4 t-\mu)\right]- \\
& -\delta_{\mathbf{n m} \pm \mathbf{a}} \delta\left(\tau_{1}-\tau_{2}\right) \hat{\tau}_{3} t+\hat{\tau}_{1} \Delta\left(\tau_{1}-\tau_{2}, \mathbf{R}_{\mathbf{n}}-\mathbf{R}_{\mathbf{m}}\right)- \\
& -\Sigma_{L}\left(\tau_{1}, \tau_{2}, \mathbf{R}_{\mathbf{n}}, \mathbf{R}_{\mathbf{m}}\right) \text {. }
\end{aligned}
$$

The disorder induced self-energy $\Sigma_{L}$ can be calculated by making an average over the disorder. We use the fully renormalized group expansion method to evaluate an approximate expression for this function. It has the following structure: $\Sigma_{L}\left(\tau_{1}, \tau_{2}, \mathbf{R}_{\mathbf{n}}, \mathbf{R}_{\mathbf{m}}\right)=\delta_{\mathbf{n m}} \Sigma_{L} \times$ $\times\left(\tau_{1}-\tau_{2}\right)$, where $\Sigma_{L}\left(\tau_{1}-\tau_{2}\right)$ in the Matsubara representation can be approximate by:

$$
\Sigma_{L}\left(\omega_{n}\right)=c \tilde{V}_{L}^{2} \rho_{N}\left[\frac{i \omega_{n}}{\mu}-i \frac{\pi}{2} \operatorname{sign}\left(\omega_{n}\right)\right]
$$

and $\rho_{N}=4 /(\pi W)$ is the normal Fermi density of states in the case $\varepsilon_{F}<W / 2$. The effective disorder potential $\tilde{V}_{L}$ in (6) is $\tilde{V}_{L}=V_{L} /\left(1+V_{L} g_{3}\right), g_{3}=$ $=\rho_{N} \ln [\mu /(W-\mu)]$ (see Appendix A, and [19], where the original derivation is presented in detail).

The self-energy $\Sigma$ in the continuum limit is:

$$
\begin{gathered}
\Sigma_{\mathbf{n m}}\left(\tau_{1}, \tau_{2}\right)=\left\langle\tau_{1}, \mathbf{R}_{\mathbf{n}}|\Sigma| \tau_{2}, \mathbf{R}_{\mathbf{m}}\right\rangle= \\
=\delta\left(\mathbf{R}_{\mathbf{n}}-\mathbf{R}_{\mathbf{m}}\right) \delta\left(\tau_{1}-\tau_{2}\right) \times \\
\times\left[\frac{i \hat{\tau}_{3}}{2} \partial_{\tau_{1}} \theta\left(\tau_{1}, \mathbf{R}_{\mathbf{n}}\right)-\frac{i}{4 m} \nabla_{\mathbf{R}_{\mathbf{n}}}^{2} \theta\left(\tau_{1}, \mathbf{R}_{\mathbf{n}}+\right.\right. \\
\left.+\frac{\hat{\tau}_{3}}{8 m}\left(\nabla_{\mathbf{R}_{\mathbf{n}}} \theta\left(\tau_{1}, \mathbf{R}_{\mathbf{n}}\right)\right)^{2}-\frac{i}{2 m} \nabla_{\mathbf{R}_{\mathbf{n}}} \theta\left(\tau_{1}, \mathbf{R}_{\mathbf{n}}\right) \nabla_{\mathbf{R}_{\mathbf{n}}}\right],
\end{gathered}
$$

where the effective fermion mass $m$ is connected with the hopping parameter $t$ in the ordinary way: $m=1 /\left(a^{2} t\right)$.

In the limit of small fluctuations of the phase of the order parameter the thermodynamic potential can be expanded in powers of the self-energy $\Sigma$ :

$\Omega(\Delta, \theta)=\int_{0}^{\beta} d \tau \int d^{2} r \frac{\Delta(\tau, \mathbf{r})^{2}}{V(\mathbf{r})}-\operatorname{Tr} \ln \mathcal{G}^{-1}+\Omega_{\mathrm{kin}}(\Delta, \theta)$,

where the kinetic part of the thermodynamic potential is

$$
\Omega_{\text {kin }}(\Delta, \theta)=\beta^{-1} \operatorname{Tr} \sum_{n=1}^{\infty} \frac{1}{n}(G \Sigma)^{n} .
$$

As a rule, it is enough to consider the thermodynamic potential up to the second order in the phase gradients $\nabla \theta$. In this case, only the terms with $n=1,2$ contribute to the kinetic part of the thermodynamical potential in (7). The kinetic ( $\nabla \theta$-dependent) part of the effective potential in this case has the following structure:

$$
\Omega_{\text {kin }}(\Delta, \theta)=\frac{J}{2} \int d^{2} r(\nabla \theta)^{2},
$$

where

$$
\begin{gathered}
J(T, \mu, \Delta(T))=\frac{1}{2} T \sum_{n=-\infty}^{\infty} \frac{d^{2} k}{(2 \pi)^{2}} \operatorname{tr}\left[\hat{\tau}_{3} \mathcal{G}_{\mathbf{k}}\left(i \omega_{n}\right) \mathrm{e}^{i \delta \omega_{n} \tau_{3}}\right]+ \\
+\frac{t^{2}}{2} T \sum_{n=-\infty}^{\infty} \frac{d^{2} k}{(2 \pi)^{2}} \mathbf{k}^{2} \operatorname{tr}\left[\mathcal{G}_{\mathbf{k}}\left(i \omega_{n}\right) \mathcal{G}_{\mathbf{k}}\left(i \omega_{n}\right)\right]
\end{gathered}
$$

(for details see [29], for example). The neutral fermion Green's function $\mathcal{G}$ in the momentum and Matsubara frequency space has the following form:

$$
\mathcal{G}_{\mathbf{k}}\left(i \omega_{n}\right)=\frac{1}{i \omega_{n}-\xi_{\mathbf{k}} \hat{\tau}_{1}-\Delta_{\mathbf{k}} \hat{\tau}_{3}-\Sigma_{L}\left(\omega_{n}\right)} .
$$

Now, having the expression for the thermodynamic potential, we are able to derive the final set of equations which define the phase diagram of the system.

\subsection{The gap equation}

In order to evaluate the equation for the mean-field critical temperature of the system above which $\Delta_{\mathbf{k}}=0$ it is necessary to minimize the potential part of the thermodynamic potential with respect to the order parameter $\Delta_{\mathbf{k}}$ : 


$$
\left.\frac{\delta \Omega}{\delta \Delta_{\mathbf{k}}}\right|_{\nabla \theta=0}=0 .
$$

This equation has the following form in the momentum space:

$$
\Delta_{\mathbf{k}}=-V \gamma_{\mathbf{k}} \int \frac{d^{2} q}{(2 \pi)^{2}} \gamma_{\mathbf{q}} T \sum_{n=-\infty}^{\infty} \operatorname{tr}\left[\hat{\tau}_{x} \mathcal{G}_{\mathbf{q}}\left(i \omega_{n}\right)\right] \equiv \gamma_{\mathbf{k}} \Delta
$$

It is seen from this equation that the order parameter has the momentum dependence supposed a priori (see above). The momentum integrals have to be performed over the region given by $\left|\xi_{\mathbf{k}}\right| \leq \varepsilon_{D}$, $\xi_{\mathbf{k}}+\mu \geq \varepsilon_{\mathrm{mob}}$. The last condition requires that the energy of the band electrons must be larger than the mobility edge energy $\varepsilon_{\mathrm{mob}}$. The value of this parameter can be estimated as $\varepsilon_{\mathrm{mob}}=\left(c / \rho_{N}\right) \ln ^{2}\left(c / c_{\mathrm{met}}\right)$, where the metalization concentration $c_{\text {met }}$ is: $c_{\text {met }}=$ $=\left|\varepsilon_{l o c}\right| \rho_{N} / 2$, which is defined by the local energy level $\varepsilon_{\text {loc }}=-\left(1 / \rho_{N}\right) /\left(\exp \left[V_{L} \rho_{N}\right]-1\right)($ see [19] for details)

The gap equation (12) can be transformed to

$$
1=\frac{V}{\alpha^{2} \pi^{2} T} \int \frac{d^{2} k}{(2 \pi)^{2}} \gamma_{\mathbf{k}}^{2} \sum_{n=-\infty}^{\infty} \frac{1}{\left[2 n+1-\Sigma_{L}^{\prime \prime} /(\alpha \pi T)\right]^{2}+E_{\mathbf{k}}^{2} /(\alpha \pi T)^{2}},
$$

where $E_{\mathbf{k}}=\sqrt{\xi_{\mathbf{k}}^{2}+\Delta_{\mathbf{k}}^{2}}, \alpha=1-c \tilde{V}_{L}^{2} \rho_{N} / \mu$, and $\Sigma_{L}^{\prime \prime} \equiv \operatorname{Im} \Sigma_{L}=-\operatorname{sign}\left(i \omega_{n}\right)(1 / 2) \pi c \tilde{V}_{L}^{2} \rho_{N}$.

The summation over the Matsubara frequencies can be formally performed in (13), and the gap equation can be written as

$$
\begin{aligned}
& 1=-\frac{i V}{4 \alpha \pi} \int \frac{d^{2} k}{(2 \pi)^{2}} \frac{\gamma_{\mathbf{k}}^{2}}{E_{\mathbf{k}}}\left[\psi\left(\frac{1}{2}+\frac{\sum_{L}^{\prime \prime}}{2 \alpha \pi T}+i \frac{E_{\mathbf{k}}}{2 \alpha \pi T}\right)+\psi\left(\frac{1}{2}-\frac{\sum_{L}^{\prime \prime}}{2 \alpha \pi T}+i \frac{E_{\mathbf{k}}}{2 \alpha \pi T}\right)-\right. \\
&\left.-\psi\left(\frac{1}{2}+\frac{\Sigma_{L}^{\prime \prime}}{2 \alpha \pi T}-i \frac{E_{\mathbf{k}}}{2 \alpha \pi T}\right)-\psi\left(\frac{1}{2}-\frac{\sum_{L}^{\prime \prime}}{2 \alpha \pi T}-i \frac{E_{\mathbf{k}}}{2 \alpha \pi T}\right)\right]
\end{aligned}
$$

where

$$
\psi(x)=-\gamma+\sum_{n=0}^{\infty}\left(\frac{1}{n+1}-\frac{1}{n+x}\right)
$$

is the di-gamma function, $\gamma=-\psi(1) \simeq 0.577$ is Euler's constant [30].

It is easy to see that equation (14) has the standard form in the clean limit:

$$
1=V \int \frac{d^{2} k}{(2 \pi)^{2}} \gamma_{\mathbf{k}}^{2} \frac{\tanh \left[E_{\mathbf{k}} /(2 T)\right]}{2 E_{\mathbf{k}}} .
$$

\subsection{The number equation}

The number equation which connects the free carrier concentration $c$ with the chemical potential $\mu$ of the system is defined by:

$$
\left.\frac{1}{v} \frac{\partial \Omega}{\partial \mu}\right|_{\nabla \theta=0}=-c
$$

where $v$ is volume of the system.

The explicit calculations give (see, for example [21]):

$$
c=T \sum_{\omega_{n}} \int \frac{d^{2} k}{(2 \pi)^{2}} \operatorname{tr}\left[\mathcal{G}_{\mathbf{k}}\left(\omega_{n}\right) \hat{\tau}_{3} \exp \left(i \delta \omega_{n} \hat{\tau}_{3}\right)\right] .
$$

In detail,

$$
c=\int \frac{d^{2} k}{(2 \pi)^{2}}\left[1-\frac{2}{\alpha^{2} \pi^{2} T} \sum_{n=-\infty}^{\infty} \frac{\xi_{\mathbf{k}}}{\left[2 n+1-\Sigma_{L}^{\prime \prime} /(\alpha \pi T)\right]^{2}+E_{\mathbf{k}}^{2} /(\alpha \pi T)^{2}}\right] .
$$

Similarly to the gap equation, the summation over $n$ can be formally performed, and the number equation can be transformed to 


$$
\begin{gathered}
c=\int \frac{d^{2} k}{(2 \pi)^{2}}\left(1+\frac{i \xi_{\mathbf{k}}}{2 \alpha \pi E_{\mathbf{k}}}\left[\psi\left(\frac{1}{2}+\frac{\Sigma_{L}^{\prime \prime}}{2 \alpha \pi T}+i \frac{E_{\mathbf{k}}}{2 \alpha \pi T}\right)+\psi\left(\frac{1}{2}-\frac{\sum_{L}^{\prime \prime}}{2 \alpha \pi T}+i \frac{E_{\mathbf{k}}}{2 \alpha \pi T}\right)-\right.\right. \\
\left.\left.-\psi\left(\frac{1}{2}+\frac{\Sigma_{L}^{\prime \prime}}{2 \alpha \pi T}-i \frac{E_{\mathbf{k}}}{2 \alpha \pi T}\right)-\psi\left(\frac{1}{2}-\frac{\Sigma_{L}^{\prime \prime}}{2 \alpha \pi T}-i \frac{E_{\mathbf{k}}}{2 \alpha \pi T}\right)\right]\right) .
\end{gathered}
$$

This equation in the clean limit also acquires the standard form:

$$
c=\int \frac{d^{2} k}{(2 \pi)^{2}}\left[1-\frac{\xi_{\mathbf{k}}}{E_{\mathbf{k}}} \tanh \left(\frac{E_{\mathbf{k}}}{2 T}\right)\right] .
$$

The carrier concentration dependence of the mean-field critical temperature $T_{c}^{M F}$ is defined by Eqs.(14) and (18) at $\Delta=0$.

\subsection{The Berezinskii-Kosterlitz-Thouless equation}

The temperature below which the phases of the order parameter in a $2 \mathrm{D}$ system with continuous symmetry become algebraically correlated is called the critical temperature of the BKT transition, in analogy with the phase transition in the 2D spin $X Y$ model. This is the only possible finite temperature phase transition in such systems.

In the case of the 2D spin $X Y$ model with the Hamiltonian $H_{X Y}=(J / 2) \sum_{\mathbf{n}, \boldsymbol{\rho}}\left(\theta_{\mathbf{n}}-\theta_{\mathbf{n}+\rho}\right)^{2}$, where $\rho$ is the nearest neighbor vector, the BKT transition critical temperature is:

$$
T_{B K T}=\frac{\pi}{2} J .
$$

In the superconducting case the equation for such a critical temperature can be obtained immediately after a comparison of the Hamiltonian $H_{X Y}$ and Eq. (19) with the kinetic part of the thermodynamic potential (8):

$$
T_{c}=\frac{\pi}{2} J\left(T_{c}, \mu, \Delta\left(T_{c}\right)\right),
$$

where the function $J\left(T_{c}, \mu, \Delta\left(T_{C}\right)\right)$, gap $\Delta\left(T_{C}\right)$, and the chemical potential $\mu$ are defined by (9), (14), and (18), respectively. Contrary to the $X Y$-model case, the BKT equation (21) is a complicated equation, since «the stiffness» $J$ depends on temperature, chemical potential, and the gap amplitude, which are also functions of temperature.

It is possible to show from (9) that the expression for $J$ is:

$$
J(T, \mu, \Delta(T))=\frac{t}{2} c-\frac{t^{2}}{\alpha^{2} \pi^{2} T} \sum_{n=-\infty}^{\infty} \int \frac{d^{2} k}{(2 \pi)^{2}} \mathbf{k}^{2} \frac{\left[2 n+1-\Sigma_{L}^{\prime \prime} /(\alpha \pi T)\right]^{2}-E_{\mathbf{k}}^{2} /(\alpha \pi T)^{2}}{\left[\left(2 n+1-\Sigma_{L}^{\prime \prime} /(\alpha \pi T)\right)^{2}+E_{\mathbf{k}}^{2} /(\alpha \pi T)^{2}\right]^{2}} .
$$

Therefore, the critical temperature equation for the doped metallic system is:

$$
T_{c}=\frac{\pi t}{4} c-\frac{t^{2}}{2 \alpha^{2} \pi T_{c}} \sum_{n=-\infty}^{\infty} \int \frac{d^{2} k}{(2 \pi)^{2}} \mathbf{k}^{2} \frac{\left[2 n+1-\Sigma_{L}^{\prime \prime} /\left(\alpha \pi T_{c}\right)\right]^{2}-E_{\mathbf{k}}^{2}\left(T_{c}\right) /\left(\alpha \pi T_{c}\right)^{2}}{\left[\left(2 n+1-\Sigma_{L}^{\prime \prime} /\left(\alpha \pi T_{c}\right)\right)^{2}+E_{\mathbf{k}}^{2}\left(T_{c}\right) /\left(\alpha \pi T_{c}\right)^{2}\right]^{2}} .
$$

Similarly to the gap and the number equations, this equation can be expressed by means of an integral over di-gamma functions:

$$
\begin{aligned}
& T_{c}=\frac{\pi t}{4} c+\frac{i t^{2}}{2 \alpha} \int \frac{d^{2} k}{(2 \pi)^{2}} \mathbf{k}^{2}\left[\frac{1}{2}+\left(\frac{E_{\mathbf{k}}}{2 \alpha \pi T}\right)^{2} \frac{\partial}{\partial E_{\mathbf{k}}^{2} /\left(2 \alpha \pi T_{c}\right)^{2}}\right]\left[\psi\left(\frac{1}{2}+\frac{\sum_{L}^{\prime \prime}}{2 \alpha \pi T_{c}}+i \frac{E_{\mathbf{k}}}{2 \alpha \pi T_{c}}\right)+\right. \\
& \left.+\psi\left(\frac{1}{2}-\frac{\Sigma_{L}^{\prime \prime}}{2 \alpha \pi T_{c}}+i \frac{E_{\mathbf{k}}}{2 \alpha \pi T_{c}}\right)-\psi\left(\frac{1}{2}+\frac{\Sigma_{L}^{\prime \prime}}{2 \alpha \pi T_{c}}-i \frac{E_{\mathbf{k}}}{2 \alpha \pi T_{c}}\right)-\psi\left(\frac{1}{2}-\frac{\Sigma_{L}^{\prime \prime}}{2 \alpha \pi T_{c}}-i \frac{E_{\mathbf{k}}}{2 \alpha \pi T_{c}}\right)\right] .
\end{aligned}
$$

In the clean limit, equation (22) transforms to:

$$
T_{c}=\frac{\pi t}{4} c-\frac{\pi t^{2}}{8 T_{c}} \int \frac{d^{2} k}{(2 \pi)^{2}} \mathbf{k}^{2} \frac{1}{\cosh ^{2}\left(E_{\mathbf{k}} /\left(2 T_{c}\right)\right)} .
$$

The carrier concentration dependence of the critical temperature can be found by solving the set of self-consistent equations (14), (18), and (22). 


\section{Solutions}

The solution of the system of equations (14) and (18) at zero value of the gap $\Delta=0$ defines the doping dependence of the mean-field critical temperature $T_{c}^{M F}$. For simplicity, we put the mobility edge $\varepsilon_{\text {mob }}$ equal to zero, and the impurity potential $\tilde{V}_{L}$ equal to $V_{L}$. This is correct when $c\left(=c_{\text {dop }}\right)$ and $V_{L}$ are not very large.

The dependencies $T_{c}^{M F}(c)$ in the case $V_{L}=0$ at different values of the attraction $V$ and $\varepsilon_{D}$ is presented in Fig. 1. The critical temperature $T_{c}^{M F}$ is extremely small at low concentrations when the coupling $V$ is small comparing to $W$ (see also [34], for example). Formally, it is caused by presence of the factor $\gamma_{\mathbf{k}}$ in the gap equation (14). The presence of this factor decreases the effective density of states, and hence the
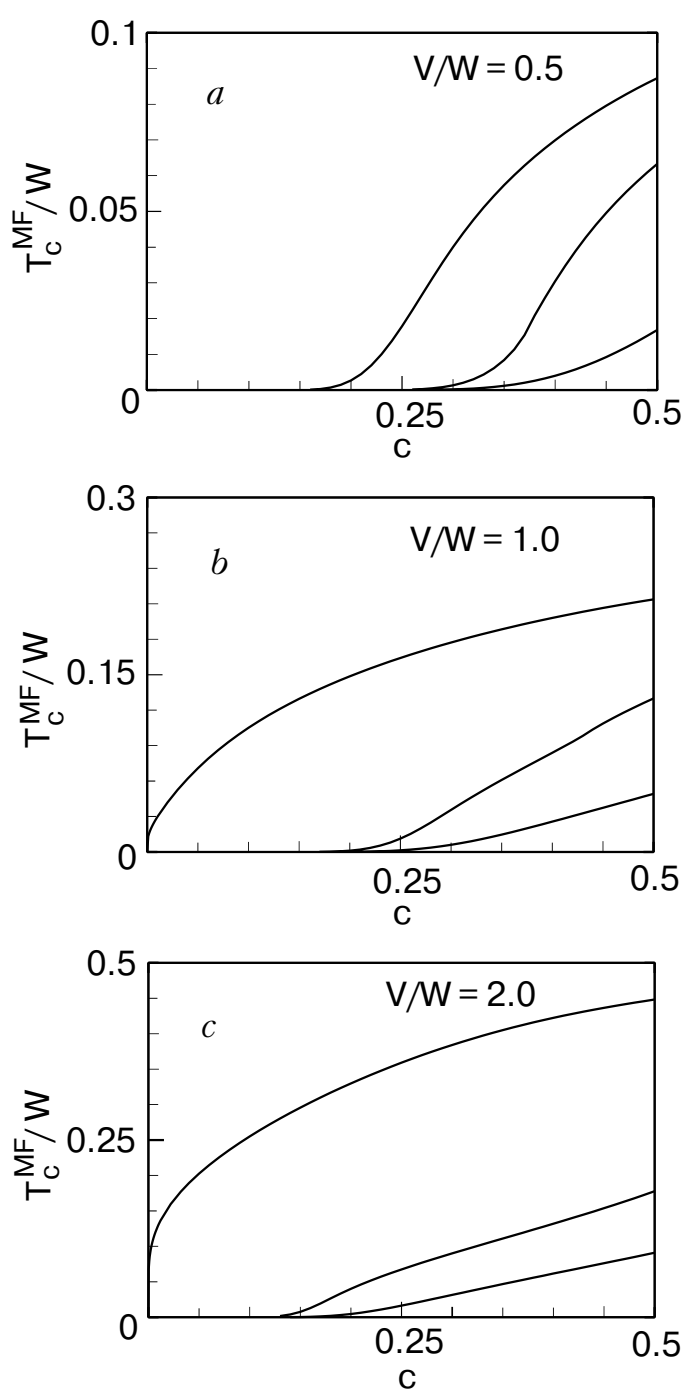

Fig. 1. The doping dependence of the mean-field critical temperature $T_{C}^{M F}$ in the case when the dopants are absent at different values of the coupling and Debye frequency. The values of the Debye frequency are $\varepsilon_{D} / W=0.125,0.25$ and 1.0 . Here and in Fig. 2 the critical temperature increases with $\varepsilon_{D}$ increasing. effective coupling at low carrier densities, since $\gamma_{\mathbf{k}}^{2} \simeq k_{F}^{2}$ is small in this case. It is interesting that the boson frequency $\varepsilon_{D}$ decreasing leads to a similar phenomena. The factor $\theta\left(\varepsilon_{D}^{2}-\xi_{\mathbf{k}}^{2}\right)$ is more important in the $d$-wave pairing channel comparing with the $s$-wave pairing case.

The same carrier density dependence of the critical temperature $T_{c}$ can be found by solving system (14), (18) and (22) (Fig. 2). It is interesting that $T_{B K T} \sim c$ at low carrier densities in the $s$-wave pairing channel at any $V$ and $\varepsilon_{D}$. This indicates the fact that the system with the $s$-wave pairing at low carrier densities is in the Bose-condensation regime. The situation is quite different in the $d$-wave pairing case. The system is in the Bose-Einstein condensation regime only, when $V$ is larger than some critical value. In fact, as it follows from Fig. 2, $T_{C} \sim c$ at low carrier densities and rather large $V$. However, this is true only when $\varepsilon_{D}$ is
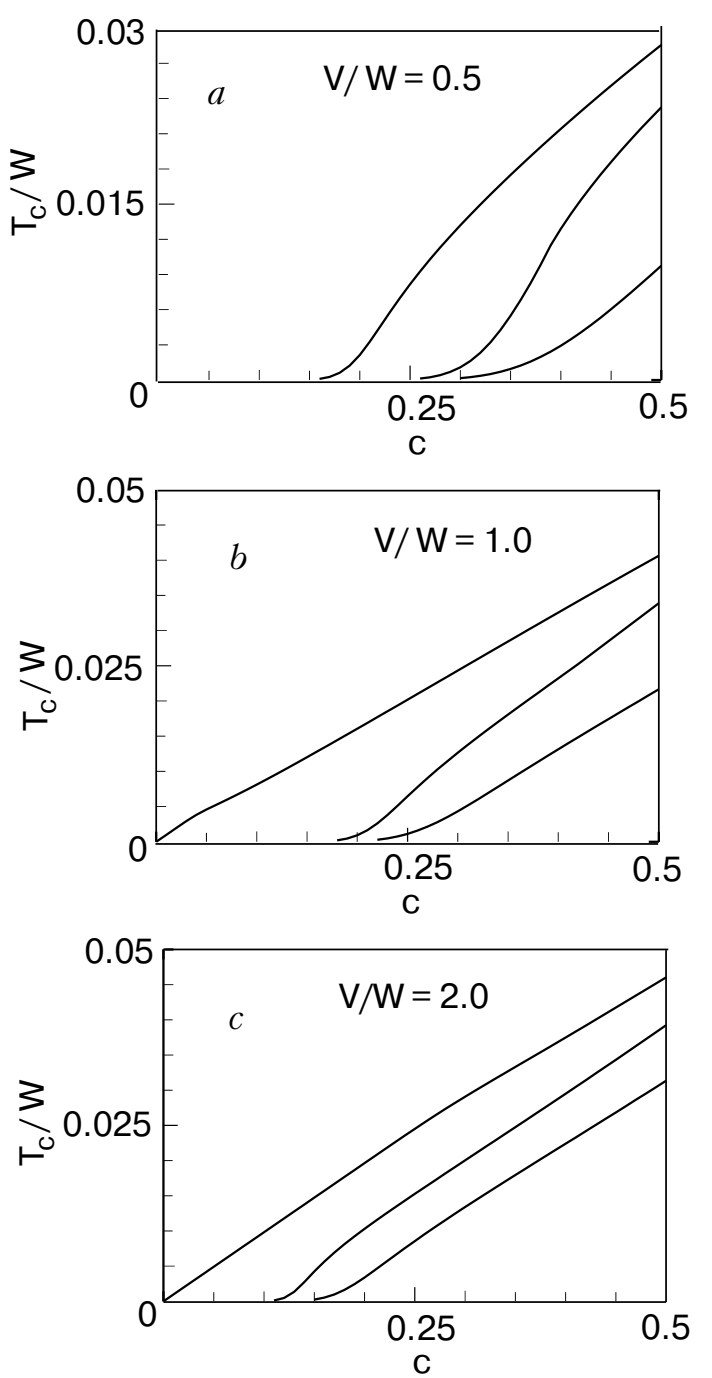

Fig. 2. The doping dependence of the superconducting critical temperature $T_{C}$ in the case, when the dopants are absent at different values of the coupling and of the Debye frequency. The values of $\varepsilon_{D} / W$ are the same as in Fig. 1. 


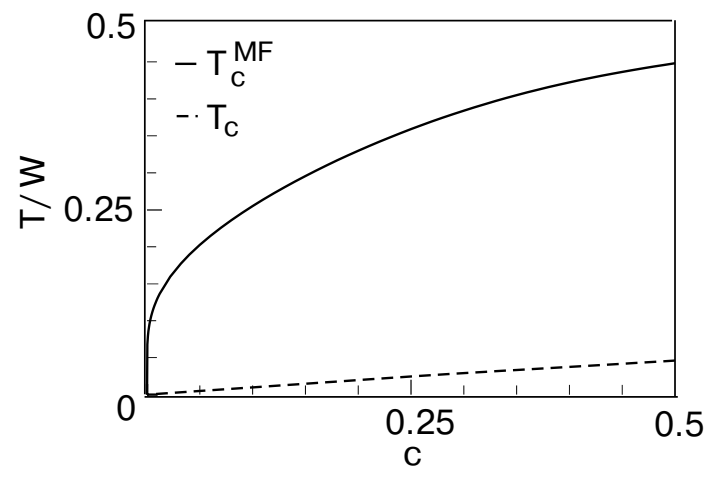

Fig. 3. The phase diagram at $V / W=2, \varepsilon_{D} / \mathrm{W}=1$ for the case when the dopants are absent.

of order or larger than $W$. In the cases of low $V$ and $\varepsilon_{D}$, the temperature $T_{C}$ becomes significantly different from zero at some finite value of $c$, which increases with $V$ and $\varepsilon_{D}$ decreasing.

In the strong coupling and large $\varepsilon_{D}$ case, the term proportional to $c$ is the largest one on the right-hand side of equation (22). In this case $T_{c} \simeq \pi t c / 4$ and it is much smaller than $T_{c}^{M F}$ (Fig. 3).

The disorder leads to a significant change of the phase diagram at large carrier densities. Namely, the curves $T_{c}^{M F}(c)$ and $T_{B K T}(c)$ decrease with the doping increasing when $V_{L}$ is large enough (Fig. 4). This provides a bell-like shape of the doping dependence of the superconducting critical temperature, similar to the
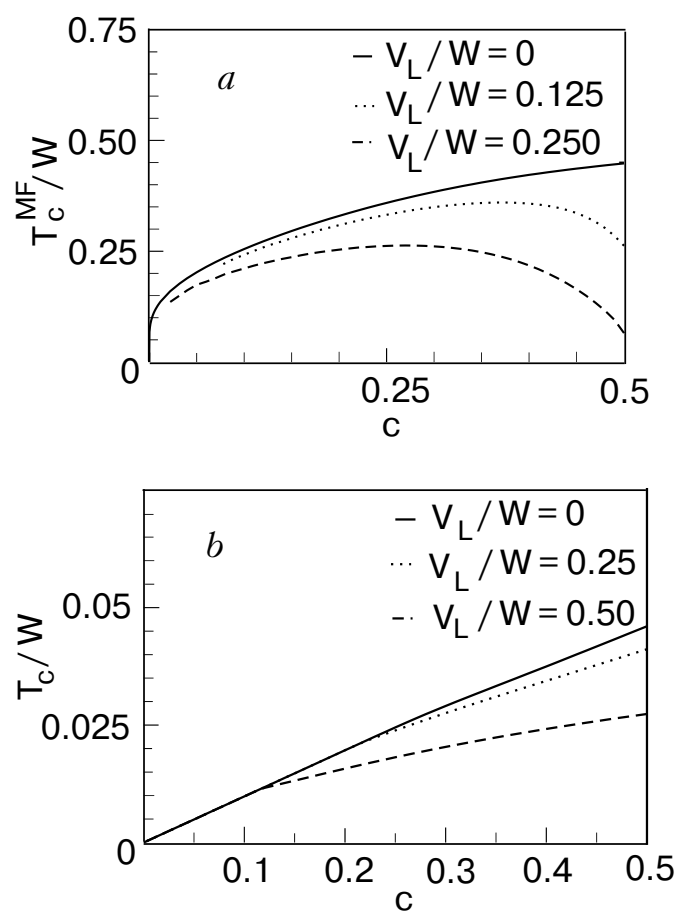

Fig. 4. The doping dependence of the mean-field critical temperature $T_{C}^{M F}$ and of the critical temperature $T_{C}$ at $V / W=2, \varepsilon_{D} / W=1$ and different values of the disorder potential. ones found experimentally in cuprates. However, we must stress that it seems problematic to reproduce quantitatively the phase diagram of HTSCs within the framework of this model. Despite the fact that the model has a phase diagram which qualitatively resembles that of HTSCs, a more complicated realistic choice of the model parameters must be considered in order to describe experimental results. In particular, the correlation effects, the realistic quasiparticle spectra and the boson dispersion relation should be taken into account more precisely.

\section{Conclusions}

To conclude, it has been shown that the dopants indeed play a twofold role in formation of the $d$-wave superconducting condensate in 2D doped metals at finite temperatures. On one hand, they are a source of metalization, supplying carriers to the system, but on the other hand, they are the centers of carrier scattering, both in free and bound states. It was shown that, similarly to the zero-temperature case, the superconductivity exists in finite range of carrier densities, provided that the disorder is large enough. The doping dependencies of the mean-field and observed critical temperatures have a bell-like shape in this case.

It was not an aim of the paper to describe quantitatively real HTSC cuprates, therefore, the problem was simplified. A self-consistent study of the dopant role in HTSCs and their influence on magnetic, electric, and superconducting subsystems requires a more specific consideration.

We would like to thank Prof. Yu.G. Pogorelov for numerous enlightening discussions. V.M.T. acknowledges support from the National Science Foundation under grant number DMR-0210717.

\section{Appendix A: Disorder average for the supercon- ducting system}

In order to find the neutral fermion Green's function averaged over disorder, let us write the Hamiltonian for neutral fermions in the momentum space:

$$
\begin{aligned}
H= & \sum_{\mathbf{k}, \sigma} \xi_{\mathbf{k}} \chi_{\mathbf{k}, \sigma}^{\dagger} \chi_{\mathbf{k}, \sigma}-\sum_{\mathbf{k}}\left[\Delta_{\mathbf{k}} \chi_{-\mathbf{k} \downarrow} \downarrow \chi_{\mathbf{k}, \uparrow}+\text { h.c. }\right]- \\
& -\frac{V_{L}}{N} \sum_{\mathbf{k}, \mathbf{k}_{1}, \mathbf{n}, \sigma} \exp \left(i\left(\mathbf{k}^{\prime}-\mathbf{k}\right) \mathbf{n}\right) \chi_{\mathbf{k}^{\prime}, \sigma}^{\dagger} \chi_{\mathbf{k}, \sigma}
\end{aligned}
$$

where $\mathbf{n}$ corresponds to the sites where a dopant is present, and the superconducting gap $\Delta_{\mathbf{k}}$ is defined self-consistently by Eqs.(14) and (18).

The retarded fermion Nambu Green's function is defined by (5). This Green's function is a $2 \times 2$ matrix 
in the Nambu space. It is important to distinguish in the equations of motion, which follow from (A.1), the diagonal and non-diagonal matrices in the Nambu $(\mathrm{N})$ and in the momentum (M) spaces. In particular, we have the following equation for the non-homogeneous system Green's function in the frequency and momentum space:

$$
\begin{gathered}
\mathcal{G}_{\mathbf{k}, \mathbf{k}^{\prime}}(\varepsilon)=\mathcal{G}_{\mathbf{k}, \mathbf{k}^{\prime}}^{(0)}(\varepsilon) \delta_{\mathbf{k}, \mathbf{k}^{\prime}}- \\
-\mathcal{G}_{\mathbf{k}, \mathbf{k}}^{(0)}(\varepsilon) \hat{V} \sum_{\mathbf{n}, \mathbf{k}^{\prime \prime}} \exp \left(i\left(\mathbf{k}-\mathbf{k}^{\prime \prime}\right) \mathbf{n}\right) \mathcal{G}_{\mathbf{k}^{\prime \prime}, \mathbf{k}^{\prime}}(\varepsilon),
\end{gathered}
$$

where the unperturbed Green's function is

$$
\mathcal{G}_{\mathbf{k}, \mathbf{k}}^{(0)}(\varepsilon)=\frac{1}{\varepsilon-\xi_{\mathbf{k}} \hat{\tau}_{3}-\Delta_{\mathbf{k}} \hat{\tau}_{1}+i 0}
$$

with $V \equiv V_{L} \hat{\tau}_{3}, \hat{\tau}_{j}$ are the Pauli matrices.

The $M$-diagonal one-particle Green's function is a self-averaged function, which can be found from (A.2) after making average over the disorder [31-33]. The general solution of (A.2) has the following form

$$
\mathcal{G}_{\mathbf{k}, \mathbf{k}}(\varepsilon)=\left\{\left[\mathcal{G}_{\mathbf{k}, \mathbf{k}}^{(0)}(\varepsilon)\right]^{-1}-\Sigma_{L \mathbf{k}}(\varepsilon)\right\}^{-1},
$$

where the self-energy part is defined by the group expansion

$$
\Sigma_{L \mathbf{k}}(\varepsilon)=-c \hat{V}(1+\mathcal{G}(\varepsilon) \hat{V})^{-1}\left[1+c \sum_{\mathbf{n} \neq 0}\left(A_{\mathbf{0} \mathbf{n}}(\varepsilon) \mathrm{e}^{-i \mathbf{k n}}+A_{\mathbf{0} \mathbf{n}}(\varepsilon) A_{\mathbf{n} \mathbf{0}}(\varepsilon)\right)\left(1-A_{\mathbf{0} \mathbf{n}}(\varepsilon) A_{\mathbf{n} \mathbf{0}}(\varepsilon)\right)^{-1}+\ldots\right]
$$

with

$$
\begin{gathered}
\mathcal{G}(\varepsilon)=\frac{1}{N} \sum_{\mathbf{k}} G_{\mathbf{k}, \mathbf{k}}(\varepsilon) ; \\
A_{\mathbf{0} \mathbf{n}}(\varepsilon)=-\hat{V} \sum_{\mathbf{k}^{\prime} \neq \mathbf{k}} \mathrm{e}^{i \mathbf{k}^{\prime} \mathbf{n}} \mathcal{G}_{\mathbf{k}^{\prime}, \mathbf{k}^{\prime}}(\varepsilon)(1+\mathcal{G}(\varepsilon) \hat{V})^{-1} .
\end{gathered}
$$

In the case, when $\Sigma_{L \mathbf{k}}(\varepsilon)$ is approximated by the first term in (A.4), the self-energy is momentum-independent:

$$
\Sigma_{L}(\varepsilon)=-c \hat{V}(1+\mathcal{G}(\varepsilon) \hat{V})^{-1} .
$$

The self-energy can be found self-consistently from the system (A.3), (A.6) and (A.5). It can be shown [19] that in the limit $W \Delta /[\pi \mu(W-\mu)] \ll 1$ and at small values of frequencies $|\varepsilon|<<\varepsilon_{D}$ the self energy is:

$$
\Sigma_{L}(\varepsilon)=c \tilde{V}_{L}^{2} \rho_{N}\left[\frac{\varepsilon}{\mu}-i \frac{\pi}{2}\right]
$$

where $\rho_{N}$ and $\tilde{V}_{L}$ are defined after Eq.(6). Equation (A.7) immediately leads to the disorder self-energy in the Matsubara frequency representation Eq. (6).

1. V.M. Loktev, Fiz. Nizk. Temp. 22, 3 (1996) [Low Temp. Phys. 22, 3 (1996)].

2. M.A. Kastner, R.J. Birgeneau, G. Shirane, and Y. Endoh, Rev. Mod. Phys. 70, 897 (1998).

3. M. Imada, A. Fujimori, and Y. Tokura, Rev. Mod. Phys. 70, 1039 (1998).

4. Yu.A. Izyumov, Phys. Usp. 42, 215 (1999).

5. Here we don't discuss the reasons and consequences for the stripes formation, or the separation of these systems on almost one-dimensional regions of metallic and dielectric phases [6-8].
6. V.M. Loktev and Yu.G. Pogorelov, Fiz. Nizk. Temp. 26, 231 (2000) [Low Temp. Phys. 26, 171 (2000)].

7. E. Dagotto, T. Hotta, and A. Moreo, Phys. Rep. 344, 1 (2001).

8. Yu.A. Izyumov and Yu.N. Skryabin, Phys. Usp. 44, 109 (2001).

9. P.W. Anderson, J. Phys. Chem. Solids 11, 26 (1959).

10. A.A. Abrikosov and L.P. Gor'kov, Sov. Phys. JETP 12, 1243 (1961).

11. P.J. Hirschfeld, P. Wölfle, and D. Einzel, Phys. Rev. B37, 83 (1988).

12. L.S. Borkowski and P.J. Hirschfeld, Phys. Rev. B49, 15404 (1994).

13. R. Fehrenbacher and M.R. Norman, Phys. Rev. B50, 3495 (1994).

14. A.I. Posazhennikova and M.V. Sadovskii, Sov. Phys. JETP Lett. 63, 358 (1996).

15. A.I. Posazhennikova and M.V. Sadovskii, Sov. Phys. JETP 85, 2124 (1997).

16. M.E. Flatte and I.M. Bayers, Solid State Phys. 52, 137 (1999).

17. V.M. Loktev and Yu.G. Pogorelov, Physica C272, 151 (1996).

18. T. Cren, D. Roditchev, W. Sacks, and J. Klein, Europhys. Lett. 54, 84 (2001).

19. V.M. Loktev and Yu.G. Pogorelov, Fiz. Nizk. Temp. 27, 1039 (2001) [Low Temp. Phys 27, 767 (2001)].

20. V.M. Loktev, Yu.G. Pogorelov, and V.M. Turkowski, Int. J. Mod. Phys. B17, 3607 (2003).

21. V.M. Loktev, R. Quick, and S.G. Sharapov, Phys. Rep. 349, 1 (2001).

22. V.P. Gusynin, V.M. Loktev, and S.G. Sharapov, JETP Lett. B65, 182 (1997).

23. K.-S. Kim, preprint cond-mat/0511527.

24. V.M. Loktev, S.G. Sharapov, and V.M. Turkowski, Physica C84, 296 (1998). 
25. V.M. Loktev and V.M. Turkowski, JETP 87, 329 (1998).

26. M.A. Ivanov, V.M. Loktev, and Yu.G. Pogorelov, Sov. Phys. JETP 74, 317 (1992).

27. In this case, the doped system is characterized by two parameters $V_{L}$ and $c$, in contrast to the one parameter Anderson model [28] with a random distribution of levels $\pm V_{L}$ over all the lattice sites.

28. P.W. Anderson, Phys. Rev. 102, 1492 (1958).

29. S.G. Sharapov, H. Beck, and V.M. Loktev, Phys. Rev. B64, 134519 (2001).
30. I.S. Gradshteyn and I.M. Ryzhyk, Tables of Integrals, Series, and Products, Academic Press, San Diego (2000).

31. I.M. Lifshitz, S.A. Gredeskul, and L.A. Pastur, Introduction to the Theory of Disordered Systems, Wiley and Sons, New York (1988).

32. M.A. Ivanov, Sov. Phys. Solid State 12, 1508 (1971).

33. M.A. Ivanov, V.M. Loktev, and Yu.G. Pogorelov, Phys. Rep. 153, 209 (1987).

34. A.M. Martin, G. Litak, B.L. Györffy, J.F. Annett, and K.I. Wysokinski, Phys. Rev. B60, 7523 (1999). 